\title{
Correction: potential therapeutic application of gold nanoparticles in B-chronic lymphocytic leukemia (BCLL): enhancing apoptosis
}

Priyabrata Mukherjee ${ }^{1,4^{*}}$, Resham Bhattacharya ${ }^{1}$, Nancy Bone ${ }^{2}$, Yean K Lee ${ }^{2}$, Chitta Ranjan Patra ${ }^{1,5}$, Shanfeng Wang ${ }^{3,4,6}$, Lichun Lu ${ }^{3,4}$, Charla Secreto ${ }^{2}$, Pataki C Banerjee ${ }^{1,7}$, Michael J Yaszemski ${ }^{3,4}$, Neil E Kay ${ }^{2}$ and Debabrata Mukhopadhyay ${ }^{1,4}$

\section{Correction}

After the publication of this work [1], it was brought to our attention that there might have been an error during compilation of the gel bands in Figure 1. We regret such unintentional errors and any inconvenience this may have caused. In order to ensure that the published figure is correct, we have repeated the experiments to provide a corrected version of Figure 1.

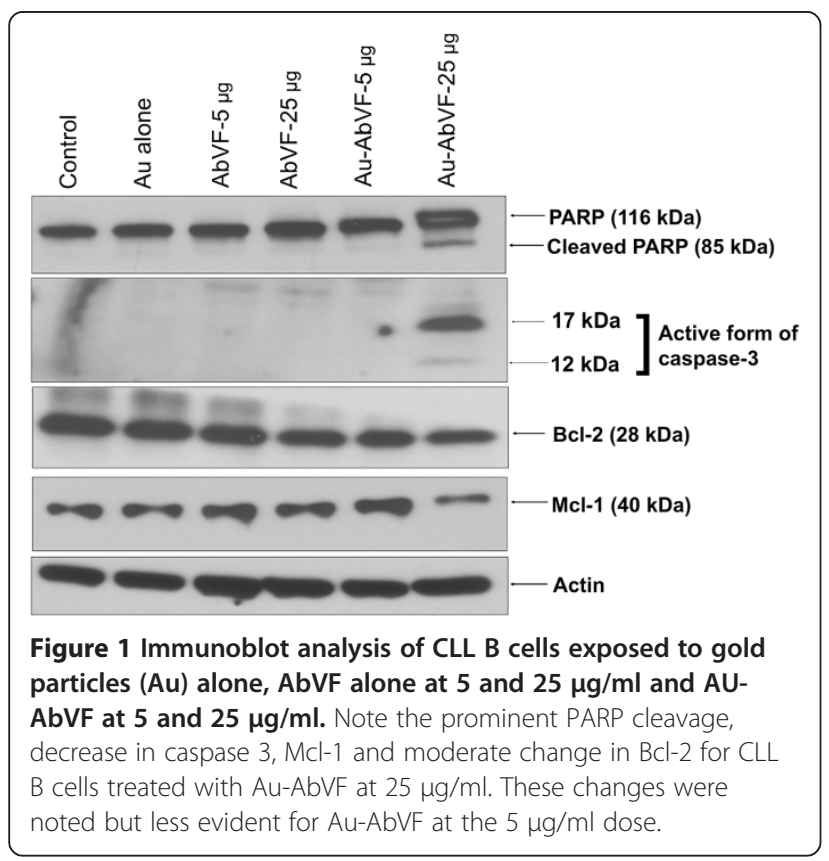

\footnotetext{
* Correspondence: mukherjee.priyabrata@mayo.edu

'Department of Biochemistry and Molecular Biology, Mayo Clinic Rochester, 200 1st Street, Rochester, MN 55905, USA

${ }^{4}$ Department of Biomedical Engineering, Mayo Clinic Rochester, 200 1st Street, Rochester, MN 55905, USA

Full list of author information is available at the end of the article
}

\section{Author details}

'Department of Biochemistry and Molecular Biology, Mayo Clinic Rochester, 200 1st Street, Rochester, MN 55905, USA. ${ }^{2}$ Department of Medicine, Division of Hematology, Mayo Clinic Rochester, 200 1st Street, Rochester, MN 55905, USA. ${ }^{3}$ Department of Orthopedic Research, Mayo Clinic, 200 1st Street, Rochester, MN 55905, USA. ${ }^{4}$ Department of Biomedical Engineering, Mayo Clinic Rochester, 200 1st Street, Rochester, MN 55905, USA. ${ }^{5}$ Current address: Department of Chemical Biology, Indian Institute of Chemical Technology [IICT], Tarnaka, Hyderabad-607, Andhra Pradesh, India. ${ }^{6}$ Current address: Department of Materials Science and Engineering, and Institute of Biomedical Engineering, The University of Tennessee, Knoxville, TN 37996-2200, USA. ${ }^{7}$ Current address: Department of Metallurgical and Material Engineering, Jadavpur University, Kolkata 700032, India.

Received: 27 June 2013 Accepted: 27 June 2013

Published: 1 July 2013

\section{Reference}

1. Potential therapeutic application of gold nanoparticles in B-chronic lymphocytic leukemia (BCLL): enhancing apoptosis. Priyabrata Mukherjee, Resham Bhattacharya, Nancy B, Lee YK, Chitta Ranjan P, Shanfeng W, Lu L, Secreto C, Banerjee PC, Yaszemski MJ, Kay NE: Debabrata Mukhopadhyay. I Nanobiotechnol 2007, 5:4

\section{doi:10.1186/1477-3155-11-23}

Cite this article as: Mukherjee et al.: Correction: potential therapeutic application of gold nanoparticles in B-chronic lymphocytic leukemia (BCLL): enhancing apoptosis. Journal of Nanobiotechnology 2013 11:23. 